\title{
A multi-dimensional FBSDE with quadratic generator and its applications
}

\author{
Eduard Rotenstein
}

\begin{abstract}
We consider, in the Markovian framework, a multi-dimensional forward - backward stochastic differential equation with quadratic growth for the generator function of the backward system. We prove an existence result of the solution and we use this result for pricing and hedging of contingent claims that depend on non-tradeable indexes by portfolios consisting in correlated risky assets.
\end{abstract}

\section{Introduction. The financial background}

In 1990, Pardoux and Peng [11] introduced the notion of nonlinear backward stochastic differential equation (for short, BSDE) and obtained existence and uniqueness result for this kind of equation. Since then, the interest in BSDEs has kept growing and there have been a lot of works on that subject. The main reason is that BSDEs are encountered in many fields of mathematics such as finance, stochastic games, optimal control, partial differential equations. This kind of stochastic systems were first studied under Lipschitz or monotonicity conditions imposed on the generator function.

A decade after, in her $\mathrm{PhD}$ thesis, Kobylanski [7] considered a new framework for such equations, which is better adapted for the study of financial markets. She considered real-valued BSDEs with quadratic growth for the coefficient. The adopted technique for proving the existence of a solution consists in some suitably exponential transformation of the equation, followed by

Key Words: Forward-backward SDEs, Quadratic growth, Financial derivatives

2010 Mathematics Subject Classification: Primary 60H10, 46L05, 91G20, 91G80

Received: December, 2013.

Revised: February, 2014.

Accepted: February, 2014. 
approximations, in a monotone manner, by Lipschitz functions, of the new generator. This approach permits the use of exponential utility functions, which role is to capture the sensitiveness with respect to risk of the investors in the market.

In the present exposition, a multidimensional forward-backward stochastic differential equation with quadratic growth for the generator function of the backward system is considered, in the Markovian framework. Namely, an existence result is established for this multidimensional equation; an application in pricing and hedging contingent claims that depend on non-tradeable indexes is presented. The hedging will be done by portfolios which consists in correlated (with respect to the non-tradable index) risky assets. This situation may appear when there exists in the financial market securities that are not the subject of transaction. For example, the choice of a company that produce kerosene (which is not tradable on a liquid market) for covering the risk caused by depreciation of the oil price is to invest in some assets that are correlated with the price of the kerosene. Therefore, to price and hedge a contingent claim (possible a multi-dimensional one) that is based on the nontradable asset, the investor can construct portfolios that contain the tradable correlated underlyings.

The paper is organized as follows. Section 2 is dedicated to the introduction of some notations and preliminary results concerning Markovian forward backward stochastic differential equations, Section 3 contains the existence result for the solution of a multi-dimensional BSDE with quadratic coefficient, while Section 4 deals with the modelization of a financial markets with tradable and non-tradable assets. Considering given a utility function for the investor, the goal consists in hedging European contingent claims within the utility maximization paradigm (see [1], [6] or [10]).

\section{Preliminaries. Notations. Hypothesis}

The purpose of this section is to introduce some basic notations and results concerning the Markovian framework of forward-backward SDEs with quadratic growth for the coefficient of the backward system, which will be needed throughout this paper. In all that follows we shall consider a finite horizon $T>0$ and a complete probability space $(\Omega, \mathcal{F}, \mathbb{P})$ on which is defined a standard $k$-dimensional Brownian motion $W=\left(W_{t}\right)_{t<T}$ whose natural filtration is denoted $\mathbb{F}=\left\{\mathcal{F}_{t}, 0 \leq t \leq T\right\}$. More precisely, $\mathbb{F}$ is the filtration generated by the process $W$ and augmented by $\mathcal{N}_{\mathbb{P}}$, the set of all $\mathbb{P}$-null sets, i.e. $\mathcal{F}_{t}=\sigma\left\{W_{s}, s \leq t\right\} \vee \mathcal{N}_{\mathbb{P}}$.

Let us consider:

(i) $\mathcal{P}$, the $\sigma$-algebra of $\mathcal{F}_{t}$-progressively measurable sets on $[0, T] \times \Omega$; 
(ii) $\mathcal{L}_{T}^{2}\left(\mathbb{R}^{d}\right)$, the set of $\mathcal{P}$-measurable and $\mathbb{R}^{d}$-valued processes $z=\left(z_{t}\right)_{t \leq T}$ such that $\int_{0}^{T}\left|z_{t}\right|^{2} d t<\infty, \mathbb{P}$-a.s.; $\mathcal{H}_{T}^{2}\left(\mathbb{R}^{d}\right)$ is the subspace of $\mathcal{L}_{T}^{2}\left(\mathbb{R}^{d}\right)$, such that $\mathbb{E}\left[\int_{0}^{T}\left|z_{t}\right|^{2} d t\right]<\infty$;

(iii) $\mathcal{S}^{2}$, the set of $\mathcal{P}$-measurable and continuous processes $Y=\left(Y_{t}\right)_{t \leq T}$ such that $\mathbb{E}\left[\sup _{t \leq T}\left|Y_{t}\right|^{2}\right]<\infty$ (this space will be also denoted by $\left.\mathcal{H}_{T}^{\infty}\left(\mathbb{R}^{\bar{d}}\right)\right)$;

Let us now introduce the four objects which define the forward backward stochastic differential system that we will consider along this paper:

1. the measurable functions $b:[0, T] \times \mathbb{R}^{n} \rightarrow \mathbb{R}^{n}$ (the drift coefficient) and $\sigma:[0, T] \times \mathbb{R}^{n} \rightarrow \mathbb{R}^{n \times k}$ (the diffusion coefficient) satisfying, $\mathbb{P}$-a.s., for $L, K>0$ :

$$
\begin{aligned}
& |b(t, x)-b(t, y)|+|\sigma(t, x)-\sigma(t, y)| \leq L|x-y| \\
& \forall(t, x, y) \in[0, T] \times \mathbb{R}^{n} \times \mathbb{R}^{n} ;
\end{aligned}
$$

the non-degeneracy condition (see Hamadène, Lepeltier, Peng [5]):

there exists a constant $C>0$ such that, for every $(t, x) \in[0, T] \times \mathbb{R}^{n}$,

$$
\frac{1}{C} I_{n} \leq \sigma(t, x) \sigma(t, x)^{t} \leq C I_{n},
$$

where $I_{n}$ represents the identity matrix of dimension $n \times n$.

2. the measurable generator function $F:[0, T] \times \mathbb{R}^{n} \times \mathbb{R}^{d} \times \mathbb{R}^{d \times k} \rightarrow \mathbb{R}^{d}$, satisfying, for some constants $\alpha>0$ and $C=\left(C_{1}, \ldots, C_{d}\right) \in\left(\mathbb{R}_{+}^{*}\right)^{d}, \mathbb{P}$-a.s.,

(i) $F(t, x, \cdot, \cdot)$ is continuous for every pair $(t, x) \in[0, T] \times \mathbb{R}^{n}$;

$$
\begin{aligned}
\left.\left|F_{i}(t, x, y, z)-C_{i}\right| z_{i}\right|^{2} \mid \leq \alpha\left(1+\left|y_{i}\right|\right), \forall i & =\overline{1, d}, \\
\forall(t, x, y, z) & \in[0, T] \times \mathbb{R}^{n} \times \mathbb{R}^{d} \times \mathbb{R}^{d \times k},
\end{aligned}
$$

by the generic $h_{i}$ understanding the $i^{t h}$ component of the vector $h$.

3. $g: \mathbb{R}^{n} \rightarrow \mathbb{R}^{d}$ is a bounded and measurable function.

Consider the pair $\left(t_{0}, x_{0}\right) \in[0, T] \times \mathbb{R}^{n}$ fixed. We will denote by $\left(X_{t}^{t_{0}, x_{0}}\right)_{t \in[0, T]}$, the solution of the forward SDE:

$$
\left\{\begin{array}{l}
d X_{t}^{t_{0}, x_{0}}=b\left(t, X_{t}^{t_{0}, x_{0}}\right) d t+\sigma\left(t, X_{t}^{t_{0}, x_{0}}\right) d W_{t}, \quad t \in\left[t_{0}, T\right], \\
X_{t}^{t_{0}, x_{0}}=x_{0}, \quad t \in\left[0, t_{0}\right] .
\end{array}\right.
$$


It is well known (see Karatzas, Shreve [8]) that, under the assumptions (H1), the SDE (1) admits a unique solution $X^{t_{0}, x_{0}}$. Moreover, for every $p \geq 2$, there exists $C_{p}>0$ such that, for all $t \in[0, T], x, x^{\prime} \in \mathbb{R}^{n}$, we have, $\mathbb{P}$-a.s.:

$$
\begin{aligned}
& \mathbb{E}\left[\sup _{s \in\left[t_{0}, T\right]}\left|X_{s}^{t_{0}, x_{0}}-X_{s}^{t_{0}, x_{0}^{\prime}}\right| p \mid \mathcal{F}_{t}\right] \leq C_{p}\left|x_{0}-x_{0}^{\prime}\right|^{p} ; \\
& \mathbb{E}\left[\sup _{s \in\left[t_{0}, T\right]}\left|X_{s}^{t_{0}, x_{0}}\right|^{p} \mid \mathcal{F}_{t}\right] \leq C_{p}\left(1+\left|x_{0}\right|^{p}\right),
\end{aligned}
$$

where the constant $C_{p}$ depends only on the Lipschitz and the linear growth constants of $b$ and $\sigma$.

We will study in Section 3 the existence of a solution for the multi-dimensional backward stochastic differential system characterized by the functions $F$ and $g$ (and denoted by QG-mFBSDE $(F, g)$ ):

$$
\left\{\begin{array}{l}
-d Y_{t}^{t_{0}, x_{0}}=F\left(t, X_{t}^{t_{0}, x_{0}}, Y_{t}^{t_{0}, x_{0}}, Z_{t}^{t_{0}, x_{0}}\right) d t-Z_{t}^{t_{0}, x_{0}} d W_{t}, \quad t \in[0, T], \\
Y_{T}^{t_{0}, x_{0}}=g\left(X_{T}^{t_{0}, x_{0}}\right),
\end{array}\right.
$$

where $X^{t, x}$ is the unique solution of Eq.(1).

Definition 1. By a solution of Eq.(2) we understand a couple

$$
\left(Y^{t_{0}, x_{0}}, Z^{t_{0}, x_{0}}\right) \in \mathcal{H}_{T}^{2}\left(\mathbb{R}^{d}\right) \times \mathcal{H}_{T}^{2}\left(\mathbb{R}^{d \times k}\right),
$$

satisfying, $\mathbb{P}$-a.s.

$Y_{t}^{t_{0}, x_{0}}=g\left(X_{T}^{t_{0}, x_{0}}\right)+\int_{t}^{T} F\left(r, X_{r}^{t_{0}, x_{0}}, Y_{r}^{t_{0}, x_{0}}, Z_{r}^{t_{0}, x_{0}}\right) d r-\int_{t}^{T} Z_{r}^{t_{0}, x_{0}} d W_{r}, \forall t \in[0, T]$.

For the case of a real-valued function $F$, we can find a complete approach in Kobylanski [7].

\section{An existence result for QG-mFBSDE}

In this Section we will provide an existence result for the solution of the BSDE (2). The main idea that will be used consists in an exponential transformation of the generator function, used on its components.

Theorem 2. Consider the functions $F:[0, T] \times \mathbb{R}^{n} \times \mathbb{R}^{d} \times \mathbb{R}^{d \times k} \rightarrow \mathbb{R}^{d}$ satisfying hypothesis (H2) and $g \in L^{\infty}\left(\mathbb{R}^{n}\right)$ which give the terminal condition of the BSDE (2), where the coefficients $b:[0, T] \times \mathbb{R}^{n} \rightarrow \mathbb{R}^{n}$ and $\sigma:[0, T] \times$ $\mathbb{R}^{n} \rightarrow \mathbb{R}^{n \times k}$ of the forward $S D E$ (1) satisfy hypothesis (H1). Then, the $Q G$ $m F B S D E(F, g)$ admits a unique solution

$$
(Y, Z) \in \mathcal{H}_{T}^{\infty}\left(\mathbb{R}^{d}\right) \times \mathcal{H}_{T}^{2}\left(\mathbb{R}^{d \times k}\right) .
$$


Proof. For every $i \in \overline{1, d}$, we consider the following exponential transformation

$$
y_{i}:=e^{2 C_{i} Y_{i}} \quad\left(\Longleftrightarrow Y_{i}=\left(2 C_{i}\right)^{-1} \ln y_{i}\right) .
$$

The obtained new generator function $f=\left(f_{i}\right)_{i=\overline{1, d}}$ is given by

$$
f_{i}(t, x, y, z):=\left\{\begin{array}{rr}
2 C_{i} y_{i} F_{i}\left(t, x, \frac{\ln y}{2 C}, \frac{z}{2 C y}\right)-\frac{\left|z_{i}\right|^{2}}{2 y_{i}}, & y \in\left(\mathbb{R}_{+}^{*}\right)^{d}, \\
0, & \text { elsewhere }
\end{array}\right.
$$

where $y=\left(y_{1}, \ldots, y_{d}\right) \in \mathbb{R}^{d}, z=\left(z_{1}, \ldots, z_{d}\right)^{t} \in \mathbb{R}^{d \times k}$ and

$$
\frac{\ln y}{2 C}:=\left(\frac{\ln y_{1}}{2 C_{1}}, \ldots, \frac{\ln y_{d}}{2 C_{d}}\right) \text { and } \quad \frac{z}{2 C y}:=\left(\frac{z_{1}}{2 C_{1} y_{1}}, \ldots, \frac{z_{d}}{2 C_{d} y_{d}}\right)^{t} .
$$

We obtain, for $y \in\left(\mathbb{R}_{+}^{*}\right)^{d}$,

$$
\begin{aligned}
f_{i}(t, x, y, z) & =2 C_{i} y_{i} F_{i}\left(t, x, \frac{\ln y}{2 C}, \frac{z}{2 C y}\right)-\frac{\left|z_{i}\right|^{2}}{2 y_{i}} \\
& \stackrel{(H 2-i i)}{\leq} 2 C_{i} y_{i}\left[\alpha\left(1+\frac{\left|\ln y_{i}\right|}{2 C_{i}}\right)+C_{i} \frac{\left|z_{i}\right|^{2}}{\left(2 C_{i} y_{i}\right)^{2}}\right]-\frac{\left|z_{i}\right|^{2}}{2 y_{i}} \\
& =\alpha y_{i}\left(2 C_{i}+\left|\ln y_{i}\right|\right) .
\end{aligned}
$$

In the same manner we obtain the left inequality, therefore

$$
\left|f_{i}(t, x, y, z)\right| \leq \alpha y_{i}\left(2 C_{i}+\left|\ln y_{i}\right|\right), \quad \forall i=\overline{1, d},
$$

hence the function $f_{i}$ is bounded provided that $y_{i}$ has the same property.

Considering that $g \in L^{\infty}\left(\mathbb{R}^{n}\right)$, we define, for each $i=\overline{1, d}, M_{i}:=e^{\alpha T}(\alpha T+$ $\left.\left\|g_{i}\right\|_{L^{\infty}}\right)$. For every $K>0$, let $\Phi_{K}:[0,1] \rightarrow \mathbb{R}, \Phi_{K} \in C^{\infty}(\mathbb{R}), \Phi_{K}=1$ on $[-K, K]$ and $\Phi_{K}=0$ on $(-K-1, K+1)^{c}$ and we consider, for each $i$, the function

$$
\varnothing_{i}(y):=\left\{\begin{aligned}
\Phi_{M_{i}}\left(\ln \frac{y}{2 C_{i}}\right), & y \geq 0 \\
0, & y<0
\end{aligned}\right.
$$

for which $\operatorname{supp}\left(\varnothing_{i}\right) \subset\left(e^{-2 C_{i}\left(M_{i}+1\right)}, e^{2 C_{i}\left(M_{i}+1\right)}\right)$.

For each $i=\overline{1, d}$, let $\hat{f}_{i}: \Omega \times[0, T] \times \mathbb{R}^{n} \times \mathbb{R}^{d} \times \mathbb{R}^{d \times k} \rightarrow \mathbb{R}$,

$$
\hat{f}_{i}(\omega, t, x, y, z):=f_{i}(\omega, t, x, y, z) \cdot \prod_{j=1}^{d} \varnothing_{j}\left(y_{j}\right),
$$


with $y_{j}$ being the $j^{\text {th }}$ component of $y$. The functions $\hat{f}_{i}$ are continuous (for every fixed $(t, x))$ and bounded since, when $y_{i}$ is bounded, then $f_{i}$ is bounded and, if $\left|y_{i}\right|$ exceeds $e^{2 C_{i}\left(M_{i}+1\right)}$, then $\hat{f}_{i}=0$. Therefore, denoting by $\hat{f}:=$ $\left(\hat{f}_{1}, \ldots, \hat{f}_{d}\right)$, from Hamadène, Hdhiri [4] or Hamadène, Lepeltier, Peng [5], there exists the pair

$$
(y, z) \in \mathcal{H}_{T}^{2}\left(\mathbb{R}^{d}\right) \times \mathcal{H}_{T}^{2}\left(\mathbb{R}^{d \times k}\right) \text { solution for } B S D E\left(\hat{f}, e^{2 C g}\right) .
$$

We have, for every $i=\overline{1, d}$, the following estimates, $\mathbb{P}$-a.s.:

$\left|\hat{f}_{i}(t, x, y, z)\right| \leq\left|f_{i}(t, x, y, z)\right| \cdot\left|\varnothing_{i}\left(y_{i}\right)\right| \cdot \underbrace{\left|\prod_{j \neq i} \varnothing_{j}\left(y_{j}\right)\right|}_{\leq 1} \leq \alpha y_{i}\left(2 C_{i}+\left|\ln y_{i}\right|\right) \cdot \varnothing_{i}\left(y_{i}\right)$,

for every $(t, x, y, z)$. The functions $h_{i}:=\alpha y_{i}\left(2 C_{i}+\left|\ln y_{i}\right|\right) \cdot \varnothing_{i}\left(y_{i}\right)$ are bounded Lipschitz functions and, since $-h_{i} \leq \hat{f}_{i} \leq h_{i}$, we obtain (by the comparison principle from El Karoui, Peng, Quenez [2]) that, for each $1 \leq i \leq d$, there exist and they are unique the pairs

$$
\left(y_{i, t}^{1}, 0\right)_{t \in[0, T]}\left(\operatorname{resp} .,\left(y_{i, t}^{2}, 0\right)_{t \in[0, T]}\right) \text { from } \mathcal{H}_{T}^{2}\left(\mathbb{R}^{d}\right) \times \mathcal{H}_{T}^{2}\left(\mathbb{R}^{d \times k}\right),
$$

solutions for the following equations with deterministic generators and terminal conditions

$$
\operatorname{BSDE}\left(-h_{i}, e^{-2 C_{i}\left\|g_{i}\right\|_{L^{\infty}}}\right)\left(\operatorname{resp} ., \operatorname{BSDE}\left(h_{i}, e^{2 C_{i}\left\|g_{i}\right\|_{L} \infty}\right)\right),
$$

solutions that verify, for every $1 \leq i \leq d$,

$$
y_{i, t}^{1} \leq y_{i, t} \leq y_{i, t}^{2}, \quad \forall t \in[0, T]
$$

Writing the second equation under differential form

$$
d y_{i, t}^{2}=-\alpha y_{i, t}^{2}\left(2 C_{i}+\left|\ln y_{i, t}^{2}\right|\right) \varnothing_{i}\left(y_{i, t}^{2}\right) d t, \quad y_{i, T}^{2}=e^{2 C_{i}\left\|g_{i}\right\|_{L^{\infty}}},
$$

we can compute its explicit solution via the following system

$$
\left\{\begin{array}{l}
d \tilde{y}_{i, t}^{2}=-\alpha \tilde{y}_{i, t}^{2}\left(2 C_{i}+\ln \tilde{y}_{i, t}^{2}\right) d t \\
\tilde{y}_{i, T}^{2}=e^{2 C_{i}\left\|g_{i}\right\|_{L} \infty}
\end{array}\right.
$$

Denoting $w_{i, t}^{2}:=\ln \tilde{y}_{i, t}^{2}$, it follows immediately that

$$
\ln \left(2 C_{i}+w_{i, t}^{2}\right)=\ln 2 C_{i}+\ln \left(1+\left\|g_{i}\right\|_{L^{\infty}}\right)
$$


equality that implies

$$
\begin{aligned}
0 & \leq \ln \tilde{y}_{i, t}^{2} \leq e^{\alpha T}\left(2 C_{i}+2 C_{i}\left\|g_{i}\right\|_{L^{\infty}}\right)-2 C_{i} \leq 2 C_{i} e^{\alpha T}\left\|g_{i}\right\|_{L^{\infty}}+2 C_{i}\left(e^{\alpha T}-1\right) \\
& \leq 2 C_{i} e^{\alpha T}\left(\alpha T+\left\|g_{i}\right\|_{L^{\infty}}\right)=2 C_{i} M_{i}
\end{aligned}
$$

from where we have that $1 \leq \tilde{y}_{i, t}^{2} \leq e^{2 C_{i} M_{i}}, \forall t \in[0, T]$. It follows that $\varnothing_{i}\left(\tilde{y}_{i, t}^{2}\right)=1, \forall t \in[0, T]$, which means that

$$
y_{i, t}^{2}=\tilde{y}_{i, t}^{2}, \quad \forall t \in[0, T] \quad \forall i=\overline{1, d} .
$$

Exactly in the same manner, for the equation with the solution $\left(y_{i, t}^{1}, 0\right)_{t \in[0, T]}$, we consider the attached system

$$
\left\{\begin{array}{l}
d \tilde{y}_{i, t}^{1}=\alpha \tilde{y}_{i, t}^{1}\left(2 C_{i}-\ln \tilde{y}_{i, t}^{1}\right) d t \\
\tilde{y}_{i, T}^{1}=e^{-2 C_{i}\left\|g_{i}\right\|_{L^{\infty}}},
\end{array}\right.
$$

we denote once again $w_{i, t}^{1}:=\ln \tilde{y}_{i, t}^{1}$ and we obtain that $e^{-2 C_{i} M_{i}} \leq \tilde{y}_{i, t}^{1} \leq 1$, which implies $\varnothing_{i}\left(\tilde{y}_{i, t}^{1}\right)=1, \forall t \in[0, T]$. Therefore,

$$
y_{i, t}^{1}=\tilde{y}_{i, t}^{1}, \quad \forall t \in[0, T], \quad \forall i=\overline{1, d} .
$$

Putting together the above estimations, we obtain, for every index $i$,

$$
e^{-2 C_{i} M_{i}} \leq y_{i, t}^{1} \leq y_{i, t} \leq y_{i, t}^{2} \leq e^{2 C_{i} M_{i}}, \quad \forall t \in[0, T]
$$

and, from the definition of $\hat{f}_{i}$, we find that $\hat{f}_{i}=f_{i}$ and $(y, z) \in \mathcal{H}_{T}^{\infty}\left(\mathbb{R}^{d}\right) \times$ $\mathcal{H}_{T}^{2}\left(\mathbb{R}^{d \times k}\right)$, i.e. $(y, z)$ is the solution of the $\operatorname{BSDE}\left(f, e^{2 C g}\right)$.

To finish the proof, we denote by

$$
Y:=\frac{\ln y}{2 C} \quad \text { and } \quad Z:=\frac{z}{2 C y} .
$$

It follows that $(Y, Z) \in \mathcal{H}_{T}^{\infty}\left(\mathbb{R}^{d}\right) \times \mathcal{H}_{T}^{2}\left(\mathbb{R}^{d \times k}\right)$. We have $Y_{T}=g\left(X_{T}^{t_{0}, x_{0}}\right)$ and, by Itô's formula, we find, for every $i=\overline{1, d}$,

$$
\begin{aligned}
& d Y_{i, t}=\frac{1}{2 C_{i} y_{i, t}} d y_{i, t}=\frac{1}{2 C_{i} e^{2 C_{i} Y_{i, t}}}\left[-f_{i}\left(t, X_{t}^{t_{0}, x_{0}}, y_{i, t}, z_{i, t}\right) d t+z_{i, t} d W_{t}\right] \\
& \quad=\frac{1}{2 C_{i} e^{2 C_{i} Y_{i, t}}}\left[-2 C_{i} e^{2 C_{i} Y_{i, t}} F_{i}\left(t, X_{t}^{t_{0}, x_{0}}, Y_{i, t}, \frac{z_{i, t}}{2 C_{i} y_{i, t}}\right) d t+\frac{z_{i, t}}{2 C_{i} y_{i, t} e^{2 C_{i} Y_{i, t}}} d W_{t}\right] \\
& \quad=-F_{i}\left(t, X_{t}^{t_{0}, x_{0}}, Y_{i, t}, Z_{i, t}\right) d t+Z_{i, t} d W_{t} .
\end{aligned}
$$

Therefore, $(Y, Z)$ is the solution of the $\mathrm{QG}-\mathrm{mFBSDE}(F, g)$. Since the uniqueness is clear, the proof is complete. 


\section{Applications}

In the Introduction we discuss a few words about non-tradable assets and hedging contingent claims by portfolios which depends on correlated tradable assets. We will consider a complete financial market characterized as follows (see Imkeller, Dos Reis, Zhang [6]). We assume that there exists a risky nontradable underlying, a risk-free account and $d$ risky assets, correlated with the non-tradable one. Consider the Brownian motion $W=\left(W_{1}, W_{2}\right)$ defined on $(\Omega, \mathcal{F}, \mathbb{P})$ and let $\tilde{W}_{1}, \ldots, \tilde{W}_{d}$ some $W_{1}$-correlated new Brownian motions, given by

$$
\tilde{W}_{i, t}:=\rho_{i} W_{1, t}+\sqrt{1-\rho_{i}^{2}} W_{2, t}, \quad \forall t \in[0, T], i=\overline{1, d} . \quad\left(\rho_{i} \in[-1,1]\right)
$$

The correlation between $\tilde{W}_{i}$ and $W_{1}$ is $\rho_{i}$, for every $1 \leq i \leq d$. We assume that the dynamics of the non-tradable index is given by

$$
d X_{t}^{0, x_{0}}=b\left(t, X_{t}^{0, x_{0}}\right) d t+\sigma\left(t, X_{t}^{0, x_{0}}\right) d W_{1, t}, \quad X_{0}^{0, x_{0}}=x_{0},
$$

where the real-valued coefficients $b$ and $\sigma$ satisfy hypothesis (H1) and the $d$ risky assets are given by

$d S_{i, t}=\alpha_{i}\left(t, X_{t}^{0, x_{0}}\right) S_{i, t} d t+\beta_{i}\left(t, X_{t}^{0, x_{0}}\right) S_{i, t} d \tilde{W}_{i, t}, S_{i, 0}=s_{i, 0}, t \in[0, T], \forall i=\overline{1, d}$

We suppose that $\alpha_{i}, \beta_{i}:[0, T] \times \mathbb{R} \rightarrow \mathbb{R}$ are bounded, measurable and strictly positive functions and we denote, for every $(t, x) \in[0, T] \times \mathbb{R}, \theta_{i}(t, x):=$ $\alpha_{i}(t, x) / \beta_{i}(t, x)$. The square integrable measurable processes $\lambda_{i}, 1 \leq i \leq d$, represents the strategy of investment in the risky asset $i$ and the sets of these admissible strategies on $[t, T]$ will be denoted by $\mathcal{A}_{i, t}$. Let $\nu_{t}$ the $\mathcal{F}_{t}$-measurable bounded r.v. - the initial investment (at time $t$ ) in the risk free account. The gain (or loss) of the investor at time $s$, obtained by investing in the risky asset $i$ is given by

$$
d G_{i, s}=\lambda_{i, s} \frac{d S_{i, s}}{S_{i, s}}, \quad G_{i, t}=0, \quad s \in[t, T], \quad \forall i=\overline{1, d} .
$$

We will hedge European contingent claims of the form $g: \mathbb{R} \rightarrow \mathbb{R}^{d}, g=$ $g\left(X_{T}^{0, x_{0}}\right)$, with $g$ a bounded and measurable function and, given a nonzero constant risk attitude parameter $\eta \in \mathbb{R}^{d}$, the investor intends to optimize the utility function $U: \mathbb{R}^{d} \rightarrow \mathbb{R}^{d}, U(x):=-\left(e^{-\eta_{1} x_{1}}, \ldots, e^{-\eta_{d} x_{d}}\right)$, where $x=\left(x_{1}, \ldots, x_{d}\right)$ (see El Karoui, Rouge [3] or Mania, Schweizer [9]). Therefore, we have

$$
V_{t}:=\sup _{\left(\lambda_{1}, \ldots \lambda_{d}\right) \in \mathcal{A}_{1, t} \times \mathcal{A}_{d, t}} \mathbb{E}\left[U\left(\nu_{t} \cdot \mathbf{1}_{d}+G_{T}+g\left(X_{T}^{0, x_{0}}\right)\right) \mid \mathcal{F}_{t}\right],
$$


where $\mathbf{1}_{d}:=(1, \ldots, 1) \in \mathbb{R}^{d}$ and $G_{T}=\left(G_{1, T}, \ldots, G_{d, T}\right)$.

A characterization of the utility $V$ given by (4) can be obtained via a QG-mFBSDE. For this, consider $\left(\mathcal{G}_{s}\right)_{s \in[0, T]}$ the natural filtration generated by the Brownian motion $W_{1}$, augmented with the set of all $\mathbb{P}$-null sets. In the perspective of Theorem 2 and of the results from [6], we have:

Proposition 3. Consider the generator function $F:[0, T] \times \mathbb{R} \times \mathbb{R}^{d} \rightarrow \mathbb{R}^{d}$, given by components:

$$
F_{i}(t, x, z):=\frac{\theta_{i}^{2}(t, x)}{2 \eta_{i}}-z_{i} \rho_{i} \theta_{i}(t, x)-\frac{\eta_{i}}{2}\left(1-\rho_{i}^{2}\right) z_{i}^{2}, \quad \forall i=\overline{1, d} .
$$

Then, the following $Q G-m F B S D E(F, g)$ :

$Y_{s}^{0, x_{0}}=g\left(X_{T}^{0, x_{0}}\right)+\int_{s}^{T} F\left(r, X_{r}^{0, x_{0}}, Z_{r}^{0, x_{0}}\right) d r-\int_{s}^{T} Z_{r}^{0, x_{0}} d\left(\mathbf{1}_{d} \cdot W_{1, r}\right), \quad s \in[0, T]$,

with $\left(X_{s}^{0, x_{0}}\right)_{s \in[0, T]}$ the solution of (3), admits a unique solution $\left(Y^{0, x_{0}}, Z^{0, x_{0}}\right) \in$ $\mathcal{H}_{T}^{\infty}\left(\mathbb{R}^{d}\right) \times \mathcal{H}_{T}^{2}\left(\mathbb{R}^{d}\right)$ such that $V_{t}=\left(V_{i, t}\right)_{i=\overline{1, d}}=\left(-e^{-\eta_{i}\left(\nu_{t}+Y_{i, t}\right)}\right)_{i=\overline{1, d}}, \mathbb{P}-$ a.s. Moreover, the investment strategy is given by

$$
\lambda_{i, s}=-\frac{\rho_{i}}{\beta_{i}\left(s, X_{s}^{0, x_{0}}\right)} Z_{i}^{0, x_{0}}+\frac{\theta_{i}\left(s, X_{s}^{0, x_{0}}\right)}{\eta_{i} \beta_{i}\left(s, X_{s}^{0, x_{0}}\right)}, \quad \forall s \leq T, \quad \forall i=\overline{1, d},
$$

where $Z^{0, x_{0}}$ is the control component from Eq.(6). This investment strategy is also the optimal point from (4).

Remark 4. It is enough to characterize the utility function $V$ defined in (4) by the solution of the $Q G-m F B S D E(F, g)$ constructed in (6) because pricing the European contingent claim $g\left(X_{T}^{0, x_{0}}\right)$ is made within the utility maximization paradigm based on the identity

$$
V_{t}\left(0, \nu_{t}\right)=: \bar{V}_{t}=\tilde{V}_{t}:=V_{t}\left(g, \nu_{t}-p_{t}\right) .
$$

According to this identity, the investor is indifferent about a portfolio with initial endowment $\nu_{t}$ without receiving one quantity of the contingent claim $g\left(X_{T}^{0, x_{0}}\right)$ and a portfolio with initial endowment $\nu_{t}-p_{t}$, but receiving one quantity of the European contingent claim; $p_{t}$ is interpreted as the time-t indifference price of the contingent claim $g\left(X_{T}^{0, x_{0}}\right)$. The indifference price does not depend on the initial endowment $\nu_{t}$.

Acknowledgements. 1. The work for this paper was supported by funds from the Project POSDRU/159/1.5/S/137750, Programe doctorale şi postdoctorale - suport pentru creşterea competitivității cercetării în domeniul Stiinţelor exacte. 
2. The author would like to express his gratitude to the anonymous referees for their comments and suggestions, which have resulted in the improvement of the presentation of this article.

\section{References}

[1] Ankirchner, S; Imkeller, P; Dos Reis, G. - Pricing and hedging of derivatives based on non-tradable underlyings, Mathematical Finance, Vol. 20, No. 2, pp. 289-312 (2010)

[2] El Karoui, N; Peng, S; Quenez, M.C. - Backward stochastic differential equations in finance, Math. Finance, 7(1), pp. 1-71 (1997)

[3] El Karoui, N.; Rouge, R. - Pricing via utility maximization and entropy, Math. Finance, 10(2), pp. 259-276 (2000)

[4] Hamadène, S.; Hdhiri I. - Backward stochastic differential equations with two distinct reflecting barriers and quadratic growth generator, Journal of Applied Mathematics and Stochastic Analysis, Vol. 2006 (Issue 1), pp. $1-28$ (2006)

[5] Hamadène, S.; Lepeltier, J.-P.; Peng, S. - BSDEs with continuous coefficients and stochastic differential games, Backward Stochastic Differential Equations, editors N. El. Karoui and L. Mazliak, Longman, Harlow, pp. 115-128 (1997)

[6] Imkeller, P; Dos Reis, G; Zhang, J - Results on numerics for FBSDE with drivers of quadratic growth, Contemporary Quantitative Finance (Essays in Honour of Eckhard Platen), pp. 159-182 (2010)

[7] Kobylanski, M. - Backward Stochastic Differential Equations and Partial Differential Equations with Quadratic Growth, The Annals of Probability, Vol. 28, No. 2, pp. 558-602 (2000)

[8] Karatzas, I.; Shreve, S.E. - Brownian motion and Stochastic Calculus, Springer-Verlag, N.Y. (1991)

[9] Mania, M; Schweizer, M. - Dynamic exponential utility indifference valuation, Ann. Appl. Probab., 15(3), pp. 2113-2143 (2005)

[10] Morlais, M.-A. - Quadratic BSDEs driven by a continuous martingale and applications to the utility maximization problem, Finance Stoch., 13(1), pp. 121-150 (2009)

[11] Pardoux, E.; Peng, S. - Solution of a Backward Stochastic Differential Equation, Systems and Control Letters, 14, pp. 55-61, (1990)

Eduard Rotenstein,

Faculty of Mathematics,

"Al. I. Cuza" University of Iaşi,

9 Carol I Blvd., 700506 Iaşi, România.

Email: eduard.rotenstein@uaic.ro 